\title{
Ibuprofen potential addition to COVID-19 early management protocols: could it be superior to paracetamol and hydroxychloroquine?
}

\author{
Mina Kelleni ${ }^{1}$ \\ ${ }^{1}$ Affiliation not available
}

May 6, 2020

\begin{abstract}
The pathogenesis of Coronavirus disease 2019 is still obscure, indeed it's only few months since it has been first reported on December 2019, yet the need for exploration of possible mechanisms, suggestion of new drugs should never be delayed. In this communication, the author proposes an integrated pathophysiological as well as pharmacological COVID-19 approach to recommend using anti-inflammatory drugs, other than glucocorticoids, suggesting ibuprofen as an example, to be added to his recently published suggested COVID-19 early management protocol. A possible explanation for the potential failure of hydroxychloroquine for COVID-19 is also explained according to the suggested concept. The author is fully aware of the ibuprofen contradictory hypothesis; as discussed and argued against in this report.
\end{abstract}

Lymphopenia has been reported to be a common feature associated with severe acute respiratory syndrome coronavirus 2 (SARS CoV-2) causing Coronavirus disease 2019 COVID-19(Chen et al., 2020; Huang et al., 2020), similarly to what has been previously reported for SARS CoV epidemic in 2002-2003(Chan \& Chen, 2008). It's currently well known that both viruses belong to the same genus of beta coronaviruses and share multiple similarities including the same ACE2 receptor to enter the target cells, as well as an immune cross reactivity(Ou et al., 2020). Further, SARS CoV-2 induced lymphopenia was recently described as an effective and reliable indicator of the severity and hospitalization in COVID-19 patients(Tan et al., 2020). Thus, if we may find a possible hypothesis to explain the lymphopenia induced by SARS CoV, it might also help us to explore a potential treatment for SARS CoV-2.

It was previously suggested that SARS CoV induced lymphopenia is likely to be caused by indirect mechanisms like an increase of cortisol levels that occurred as part of the body stress response to this severe respiratory viral infection or by an iatrogenic effect of glucocorticoids used to manage those patients. Further, cortisol level was demonstrated to be significantly higher in the lymphopenic compared with nonlymphopenic patients as well as to be significantly negatively correlated with monocytes and suppressor $\mathrm{CD} 8^{+}$cells, B lymphocytes and helper CD4 ${ }^{+}$cells in SARS CoV patients(Chan \& Chen, 2008; Panesar, Lam, Chan, Wong \& Sung, 2004). Interestingly, the adrenal gland was previously shown to express ACE2 receptors and SARS $\mathrm{CoV}$ related inflammation of various organs including the adrenal gland have been also reported. Similarly, SARS CoV has been demonstrated to cause architectural disruption and lymphocyte depletion in the spleen and lymph nodes despite no or trace ACE2 expression in examined human tissues specimens and SARS CoV has also been associated with adrenal glands' necrosis and infiltration of monocytes and lymphocytes (Hamming, Timens, Bulthuis, Lely, Navis \& van Goor, 2004; Harmer, Gilbert, Borman \& Clark, 2002). Relying on the previous mechanisms related to SARS CoV, to be added to our current knowledge that COVID-19 pathogenesis has been showed to be associated with excessive chemokines release; a cytokine 
storm that has been associated with unfavorable clinical outcomes, including some chemokines attracting the lymphocytes and other inflammatory cells to the infected tissues(Pedersen \& Ho, 2020; Xiong et al., 2020). The author suggests that SARS CoV-2, like SARS CoV may progressively cause, both directly and indirectly, a fulminant inflammation in different body organs and some of these released chemokine, and perhaps others yet to be discovered, are well known to attract the lymphocytes to those organs causing the lymphopenia encountered with COVID-19. More importantly, the author suggests that this lymphopenia might be considered as a symbol for distraction of lymphocytes to multiple organs instead of being mainly directed to the lungs, the main target organ of COVID-19. Interestingly, it might also be considered as another reflection to the previously described disturbance of the homeostasis of the interferons' immune response in COVID-19 patients(Pedersen \& Ho, 2020; Sallard, Lescure, Yazdanpanah, Mentre \& PeifferSmadja, 2020; Zhang et al., 2020). Noteworthy, hydroxychloroquine has been previously reported to cause lymphopenia and increase human immunodeficiency virus, viral load which was suggested to be only explained by a biological effect of the drug(Paton et al., 2012) and a potential likewise relationship with SARS CoV-2 induced lymphopenia should be carefully and thoroughly investigated as it might reason for its recent report of inefficacy(Magagnoli et al., 2020), especially if similar confirmatory results are released. Moreover, acute inflammation of the adrenal glands is increasing cortisol secretion, especially early in the COVID-19 clinical course, which is further augmenting the lymphopenia and might complete the unfortunate vicious circle. Noteworthy, short-duration, high-dose glucocorticoid therapy wasn't proved effective for early acute respiratory distress syndrome or for severe sepsis and when combined with mineralocorticoids, they've only shown some benefits in some selected critical cases classified with a poor prognosis, and moderate doses of glucocorticoids were suggested to be beneficial only for patients with late acute respiratory distress syndrome (Thompson, 2003). Further, it's been recommended for patients with rheumatic disease on glucocorticoids therapy to use the minimum possible doses of glucocorticoids during COVID-19 infection(Misra, Agarwal, Gasparyan \& Zimba, 2020). Thus, the author would like to agree with the clinical recommendation against the routine use of glucocorticoids in the management of COVID-19 and to confirm they should be only discussed to be administered on a case by case basis(SRLF-SFAR-SFMU-GFRUP-SPILF-SPLF, 2020, April 7) and the author would like to dispute with some other contradictory reports and encourages more colleagues to present properly performed clinical data to end any remaining controversy(Zhang et al., 2020). Most importantly, basing on the suggested pathogenesis of COVID-19 described in this manuscript, the author suggests that using anti-inflammatory drugs, other than glucocorticoids, e.g. ibuprofen might prove beneficial for early management of COVID-19 trying to ameliorate the suggested inflammatory process leading to lymphopenia and immunosuppression. Similarly, this recommendation might be considered, to be applied as early as possible during the clinical course of COVID-19 and the author suggests it might be added to his newly suggested nitazoxanide/azithromycin protocol for early management of COVID-19 (Kelleni, 2020) but in a separate arm for nitazoxanide/azithromycin/ibuprofen as well as to add it to any other already adopted protocol for early cases of COVID-19 and compare the results against the already used analgesic/antipyretic paracetamol. If proved right, it would be more superior to the currently used analgesic antipyretic paracetamol, improving the survival rate and immune response of COVID-19 patients. The author is fully aware that his hypothesis is contradictory to another one that has been suggested(Fang, Karakiulakis \& Roth, 2020), The author has also prepared a full paper disputing the contradictory hypothesis and is looking for a potential peer review. It's noteworthy to mention that ibuprofen has been previously hypothesized to be hazardous in another medical argument and later on it was proved incorrect(Sodhi \& Etminan, 2020) and it's also worthwhile to notice that some recent clinical results related to other drugs, mentioned with ibuprofen in that contradictory hypothesis, have recently revoked their claimed hazards(Bean et al., 2020). The main difference here, is that we can't wait for long time before testing a possibility that we've a beneficial drug like ibuprofen that might help us in this very depressive COVID-19 crisis, we should learn from unintended mistakes and make sure not to be repeated, the author perhaps is doing a mistake but this is something that would be only answered by our fellow researchers who might consider his as well as others claims.

Conflict of interests: None.

Funding: None 


\section{References:}

Bean D, Kraljevic Z, Searle T, Bendayan R, Pickles A, Folarin A, et al. (2020). Treatment with ACEinhibitors is associated with less severe disease with SARS-Covid-19 infection in a multi-site UK acute Hospital Trust. medRxiv: 2020.2004.2007.20056788.

Chan PK, \& Chen GG (2008). Mechanisms of lymphocyte loss in SARS coronavirus infection. Hong Kong Med J 14 Suppl 4: 21-26.

Chen T, Wu D, Chen H, Yan W, Yang D, Chen G, et al. (2020). Clinical characteristics of 113 deceased patients with coronavirus disease 2019: retrospective study. BMJ 368: m1091.

Fang L, Karakiulakis G, \& Roth M (2020). Are patients with hypertension and diabetes mellitus at increased risk for COVID-19 infection? The Lancet Respiratory Medicine 8: e21.

Hamming I, Timens W, Bulthuis MLC, Lely AT, Navis GJ, \& van Goor H (2004). Tissue distribution of ACE2 protein, the functional receptor for SARS coronavirus. A first step in understanding SARS pathogenesis. The Journal of Pathology 203: 631-637.

Harmer D, Gilbert M, Borman R, \& Clark KL (2002). Quantitative mRNA expression profiling of ACE 2, a novel homologue of angiotensin converting enzyme. FEBS Letters 532: 107-110.

Huang C, Wang Y, Li X, Ren L, Zhao J, Hu Y, et al. (2020). Clinical features of patients infected with 2019 novel coronavirus in Wuhan, China. Lancet 395: 497-506.

Kelleni MT (2020). Nitazoxanide/Azithromycin combination for COVID-19: A suggested new protocol for COVID-19 early management. Pharmacological Research: 104874.

Magagnoli J, Narendran S, Pereira F, Cummings T, Hardin JW, Sutton SS, et al. (2020). Outcomes of hydroxychloroquine usage in United States veterans hospitalized with Covid-19. medRxiv:2020.2004.2016.20065920.

Misra DP, Agarwal V, Gasparyan AY, \& Zimba O (2020). Rheumatologists' perspective on coronavirus disease 19 (COVID-19) and potential therapeutic targets. Clin Rheumatol.

Ou X, Liu Y, Lei X, Li P, Mi D, Ren L, et al. (2020). Characterization of spike glycoprotein of SARS-CoV-2 on virus entry and its immune cross-reactivity with SARS-CoV. Nature Communications 11: 1620.

Panesar NS, Lam CWK, Chan MHM, Wong CK, \& Sung JJY (2004). Lymphopenia and neutrophilia in SARS are related to the prevailing serum cortisol. European Journal of Clinical Investigation 34: 382-384.

Paton NI, Goodall RL, Dunn DT, Franzen S, Collaco-Moraes Y, Gazzard BG, et al. (2012). Effects of hydroxychloroquine on immune activation and disease progression among HIV-infected patients not receiving antiretroviral therapy: a randomized controlled trial. JAMA 308: 353-361.

Pedersen SF, \& Ho YC (2020). SARS-CoV-2: a storm is raging. J Clin Invest.

Sallard E, Lescure F-X, Yazdanpanah Y, Mentre F, \& Peiffer-Smadja N (2020). Type 1 interferons as a potential treatment against COVID-19. Antiviral research 178: 104791-104791.

Sodhi M, \& Etminan M (2020). Safety of Ibuprofen in Patients With COVID-19: Causal or Confounded? Chest:S0012-3692(0020)30572-30579.

[Recommendations of experts relating to the care in resuscitation of SARS-CoV2 epidemic patients]

[Online] Available from https://sfar.org/download/recommandations-dexpertsportant-sur-la-prise-en-charge-en-reanimation-des-patients-en-periode-depidemie-a-sars-

cov2 $/$ ? wpdmdl $=25387 \&$ refresh $=5$ e9db5d4aef411587394004. [Accessed].

Tan L, Wang Q, Zhang D, Ding J, Huang Q, Tang Y-Q, et al. (2020). Lymphopenia predicts disease severity of COVID-19: a descriptive and predictive study. Signal transduction and targeted therapy 5:33-33. 
Thompson BT (2003). Glucocorticoids and acute lung injury. Crit Care Med 31: S253-257.

Xiong Y, Liu Y, Cao L, Wang D, Guo M, Jiang A, et al. (2020). Transcriptomic characteristics of bronchoalveolar lavage fluid and peripheral blood mononuclear cells in COVID-19 patients. Emerg Microbes Infect 9: 761-770.

Zhang W, Zhao Y, Zhang F, Wang Q, Li T, Liu Z, et al. (2020). The use of anti-inflammatory drugs in the treatment of people with severe coronavirus disease 2019 (COVID-19): The Perspectives of clinical immunologists from China. Clinical immunology (Orlando, Fla) 214: 108393-108393. 